\title{
Baculoviral mid-gut gland necrosis (BMN) of kuruma shrimp (Penaeus japonicus) larvae in Japanese intensive culture systems
}

\author{
T. Sano ${ }^{1}$, T. Nishimura ${ }^{1}$, H. Fukuda ${ }^{1}$, T. Hayashida ${ }^{1}$ \& \\ K. Momoyama ${ }^{2}$ \\ ${ }^{1}$ Laboratory of Fish Pathology, Tokyo University of Fisheries; 4-5-7 Konan, Minato-ku, \\ Tokyo 108, Japan \\ ${ }^{2}$ Naikai Fisheries Laboratory; 437-77 Futashima, Aio, \\ Yamaguchi, Yamaguchi Prefecture 759-41, Japan
}

\begin{abstract}
In many shrimp farms in the Kyushu and Chugoku areas of Japan, the so-called midgut gland cloudy disease of kuruma shrimp larvae (Penaeus japonicus) has occurred since 1971. The pathological changes associated with this baculoviral mid-gut gland necrosis (BMN) are extensive cellular necrosis, collapse of mid-gut gland cells, nuclear hypertrophy and finally karyorrhexis. Electron microscopic examination revealed the presence of virions and virogenic stages in the affected nuclei. Average length and diameter of the virions detected was 310 and $72 \mathrm{~nm}$, respectively; nucleocapsids were $250 \mathrm{~nm}$ in size. Virions enclosing 2 nucleocapsids within a single envelope were rarely found. The spirally arranged capsomeres were at an angle of 37 to $38^{\circ}$ to a horizontal line meeting at right angles with the long axis of the virion. Infectivity trials resulted in high mortality of healthy mysis and juveniles (2nd post-larval stage). Juveniles at the 9th post-larval stage showed no mortality, although they could be infected easily by the agent. Hypertrophied nuclei in squashed and stained preparations of the affected gland cells can be considered to be of reliable presumptive diagnostic character, and fluorescent antibody staining can be employed to confirm the diagnosis of BMN.
\end{abstract}

\section{INTRODUCTION}

In many shrimp farms in the Kyushu and Chugoku areas of Japan, the so-called midgut gland cloudy disease of kuruma shrimp larvae, Penaeus japonicus, has occurred from May to September, the growing season, almost every year since 1971. It has drastically produced high mortality and showed fully advanced necrosis of the mid-gut gland. However, nobody has attempted to find the causative agent of this disease thus far. We had an opportunity to perform pathological examinations of the diseased shrimp in the summer of 1977 and infectivity experiments in the summers of 1978, 1980 and 1982. Consequently, we were able to obtain evidence that all moribund shrimp larvae suffered from an infection accompanied by the presence of numerous rod-shaped particles, Baculovirus virions. We designated this epizootic baculoviral "mid-gut gland necrosis" (BMN) in 1981. Characteristic changes in the nuclei of the gland cells, and the results of 
infectivity trials indicated that the baculovirus is probably the causative agent of this epizootic.

Considering that this baculovirus has the potential to produce high mortality in shrimp larvae, we tried to develop a rapid diagnostic technique including confirmative diagnosis.

\section{MATERIALS AND METHODS}

The first materials studied were moribund shrimp larvae, showing the same symptoms as shrimp in the epizootic (Fig. 1), and healthy larvae brought to the Laboratory of

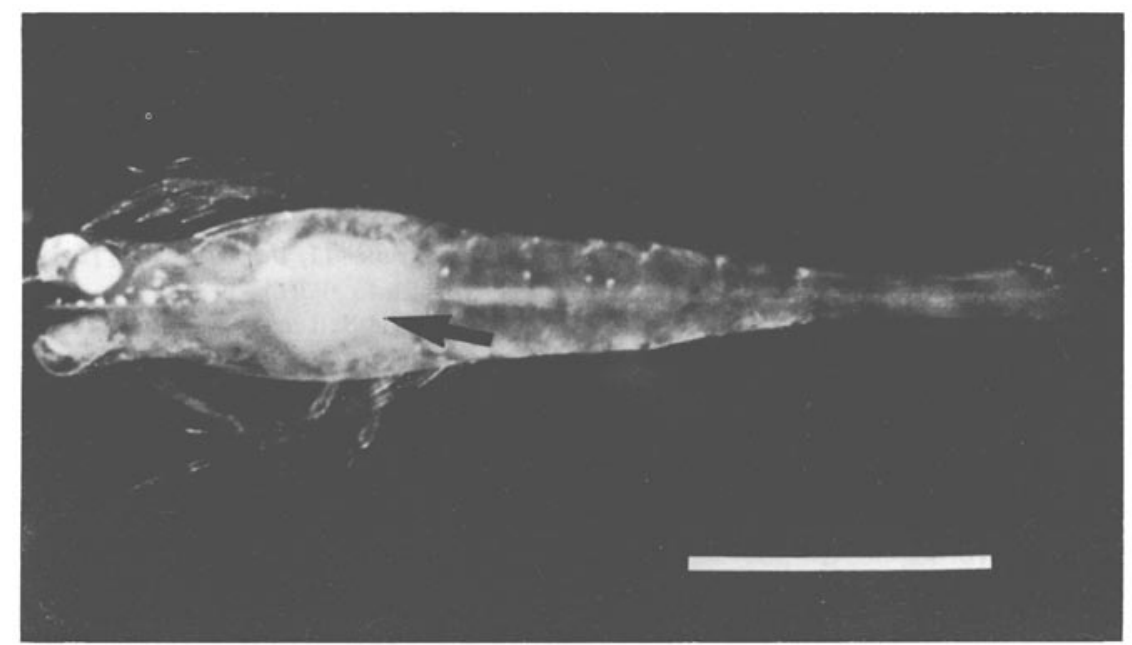

Fig. 1. General appearance of kuruma shrimp larva suffering from baculoviral mid-gut gland necrosis (BMN). Mid-gut gland (arrow) became cloudy due to severe necrosis of the organ. Bar $=2 \mathrm{~mm}$

Fish Pathology, Tokyo University of Fisheries, from a shrimp-culture farm located at Ube, Yamaguchi Prefecture (June, 1977). Materials at the 9th post-larval stage (healthy) were transported to the same laboratory from Shizuoka Prefectural Fish Farming, Numazu, Shizuoka Pref. (September, 1982).

Material for conventional microscopy was fixed in neutral $10 \%$ formalin and Bouin's solution. Sectioned preparations were stained with hematoxylin and eosin, and by VagoAmargier's method (1963). For electron microscopy, the same materials were fixed for $2 \mathrm{~h}$ in cold $2.5 \%$ glutaraldehyde (in $0.1 \mathrm{M}$ PBS adjusted to PH 7.4), rinsed for $1 \mathrm{~h}$ with $7 \%$ sucrose (in $0.1 \mathrm{M}$ PBS) and postfixed for $1 \mathrm{~h}$ with $1 \%$ osmium tetroxide (in marine crab saline). These materials were dehydrated in alcohol, embedded in epoxy resin, sectioned and stained with uranyl acetate, with subsequent staining with lead citrate, and observed with a JEM-100B electron microscope.

For electron microscopy of negative stain, the following procedures were employed: $1 \mathrm{~g}$ affected shrimp frozen at $-80^{\circ} \mathrm{C}$ for $1 \mathrm{yr}$ was homogenized while chilling, suspended in $30 \mathrm{ml}$ minimum essential medium supplemented with $2 \%$ fetal bovine serum (MEM-2), 
centrifuged at $2000 \mathrm{xg}$ for $20 \mathrm{~min}$; the supernatant was then centrifuged at $17,500 \mathrm{xg}$ for $30 \mathrm{~min}$. The precipitation was resuspended in $2 \mathrm{ml}$ Tris- $\mathrm{HCl}$ Buffer (PH 7.4, $10 \mathrm{mM}$ ). It was then negatively stained with $3 \%$ uranyl acetate and observed with a JEM-100B.

Infectivity experiments were carried out using oral and waterborne inoculation. Thirty larvae from the 2nd day post-larval stage were used for the oral inoculation test, each larva being released into a $100 \mathrm{ml}$ flask. Ten larvae (also one per flask) were used as controls. Each larva in the oral inoculation group was fed mid-gut gland removed from one diseased shrimp. Control shrimp were fed nauplius of brine shrimp. Water temperature in flask during the experiment ranged from 23.2 to $27.0^{\circ} \mathrm{C}$. The waterborne inoculation tests utilized 50 larvae from mysis II or III stage. These larvae were released into 51 jars, for control and test purposes. The inoculant was the supernatant fluid obtained by centrifuging $0.3 \mathrm{~g}$ homogenized diseased shrimp, previously frozen at $-80^{\circ} \mathrm{C}$, in $10 \mathrm{ml}$ sterilized seawater at $300 \mathrm{xg}$ for $10 \mathrm{~min}$. The supernatant fluid $(6.5 \mathrm{ml})$ was filtered through a $450 \mathrm{~nm}$ filter prior to addition to the test jar. Water temperature during this experiment ranged from 25.6 to $26.0^{\circ} \mathrm{C}$. Both control and test shrimp were fed nauplius of brine shrimp.

Waterborne inoculation of juveniles at the 9th post-larval stage was performed as follows: $1 \mathrm{~g}$ diseased larvae frozen at $-80^{\circ} \mathrm{C}$ was thawed and homogenized in $69 \mathrm{ml}$ sterilized seawater, subsequently centrifuged at $700 \mathrm{xg}$ for $10 \mathrm{~min}$. The supernatant fluid $(43 \mathrm{ml})$ was filtered through a $450 \mathrm{~nm}$ filter prior to addition to a $11 \mathrm{jar}$ in which 100 larvae were subjected to oral inoculation for $2 \mathrm{~h}$. These post-inoculation larvae were raised in $15 \mathrm{l}$ seawater at $22.5-25.0^{\circ} \mathrm{C}$.

Squashed and stained preparations of spontaneously affected mid-gut gland were checked for the presumptive diagnostic technique. The indirect immunofluorescence technique was also examined to learn whether this procedure can be employed to confirm the diagnosis, using waterborne-infected juveniles from the 9 th post-larval stage. Samples for this experiment were taken at $18,24,36,48,60,72,96$ and $120 \mathrm{~h}$ postinoculation. Squashed preparations from the glands of these samples were provided, fixed with acetone for $10 \mathrm{~min}$ at room temperature and then stained with the indirect fluorescent antibody technique using FITC-Protein A (Pharmacia Fine Chemicals).

\section{RESULTS}

The field report from the shrimp-culture farm at Aio, Yamaguchi Prefecture, on mortality caused by an epizootic indicated that the shrimp larvae suffered high mortality; $20 \%$ up to mysis or the 1st post-larval stage; $50 \%$ up to the 7 th stage (Fig. 1) and $90 \%$ up to 8 th or 9 th stage.

Light and electron microscopic examination showed no evidence of any polyhedral inclusion bodies like those produced by some insect baculoviruses in material of the affected gland of moribund larvae (Figs 2and 6). However, such preparations revealed remarkable cellular necrosis and collapse of mid-gut gland. Preparations stained with hematoxylin and eosin showed that nuclear hypertrophy and collapse always followed these cellular changes. The dimensions of the hypertrophied nuclei of such cells were $10-14 \times 12-16 \mu \mathrm{m}$, while comparable figures for normal nuclei were only $4-6 \times 4-8 \mu \mathrm{m}$. There was also no evidence of any inclusion bodies in the preparations stained according to Vago-Amargier's method (1963). 


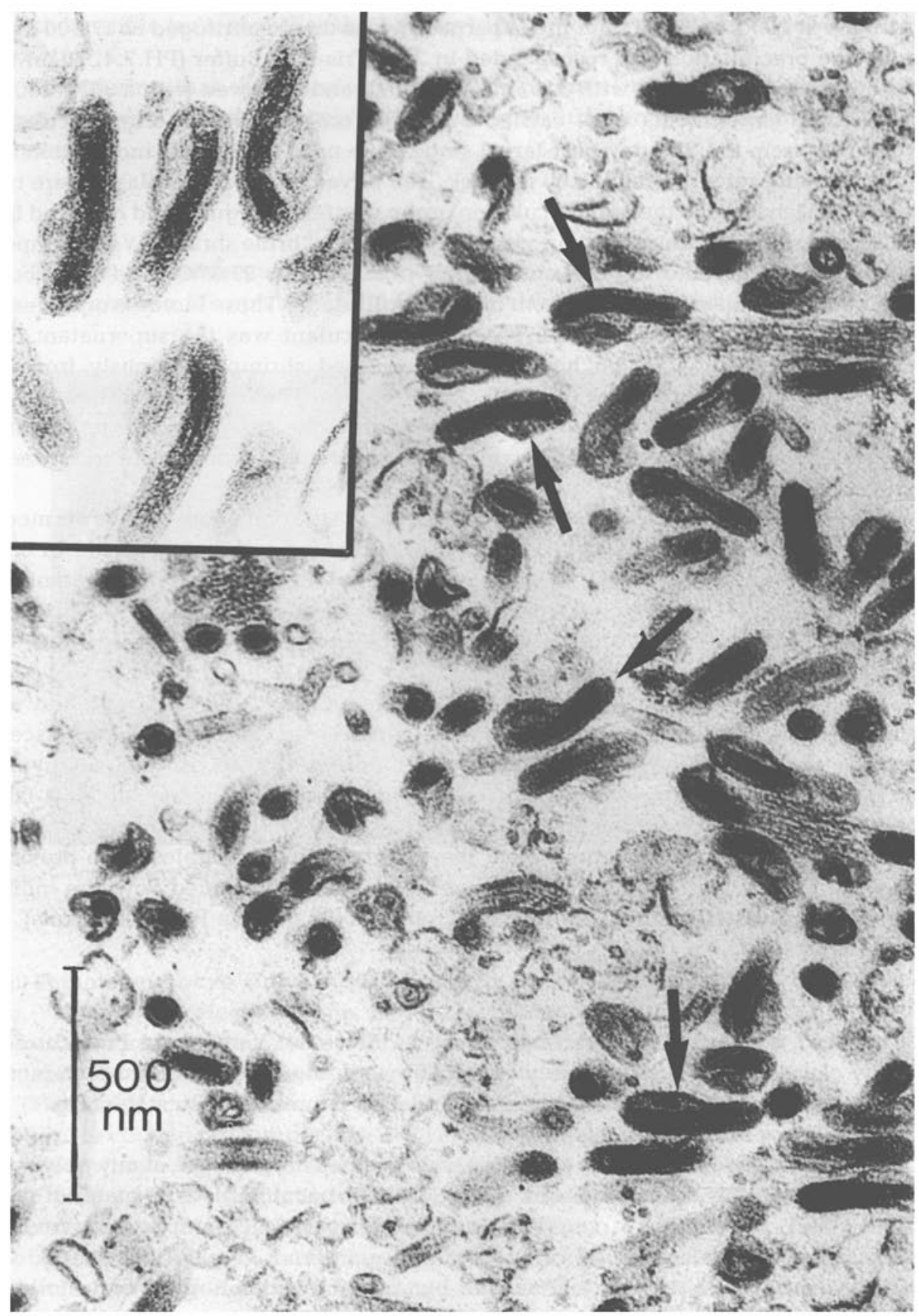

Fig. 2. Several profiles of viral particles revealing the characteristics of Baculovirus and incomplete particles representing virogenic stages in the affected nucleus: note rod-shaped virion (arrow); virions enveloped twice by outer and inner membrane (insert); virions with convex envelope, particles without core and with opened envelope, and fibrillar structure 


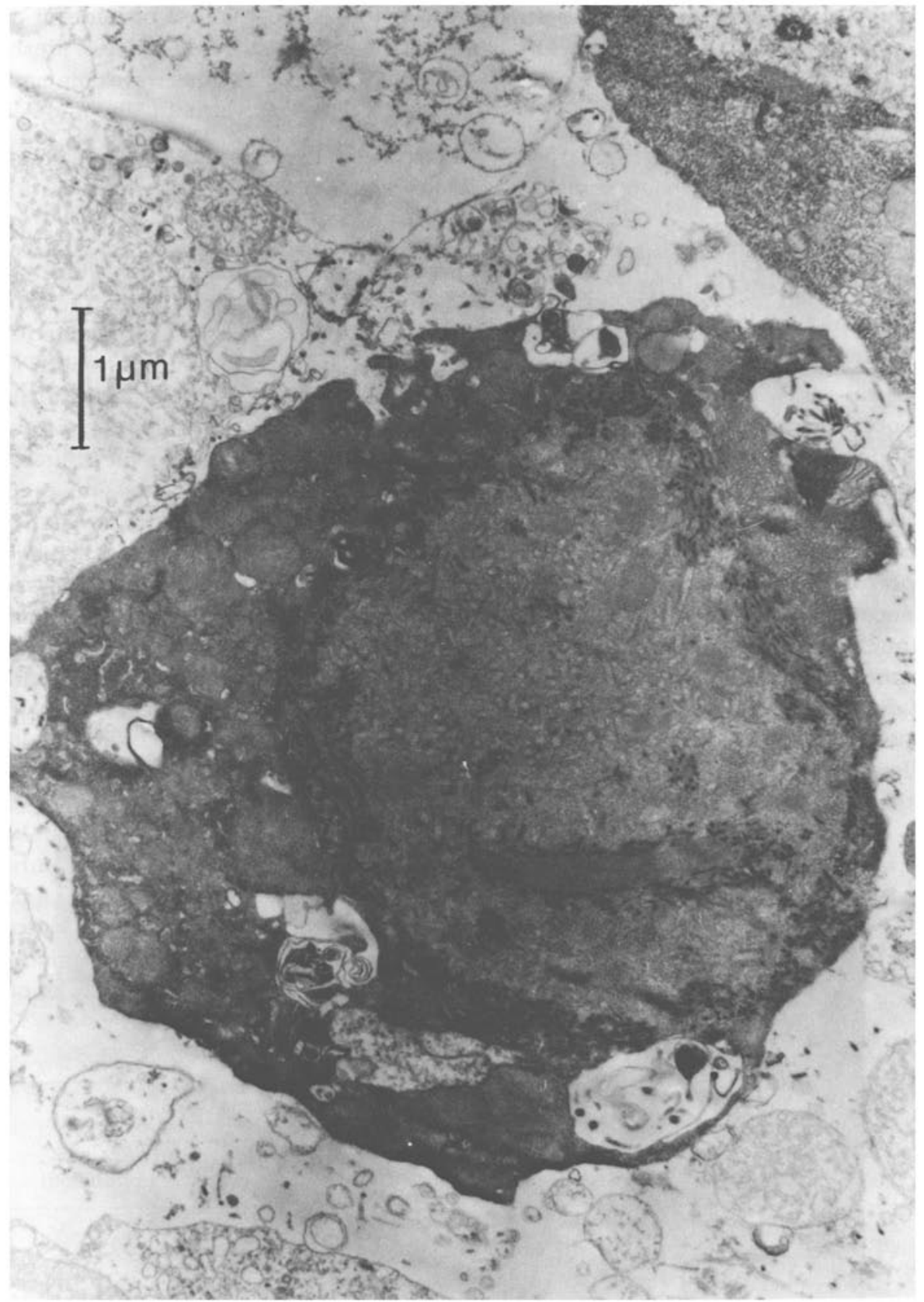

Fig. 3. Electron micrograph of an ultrathin section of mid-gut gland taken from artificially infected shrimp larvae. Note the hypertrophied nucleus and numerous baculoviruses 
Electron microscopic observations showed cytoplasmic collapse of mid-gut gland cells and nuclear hypertrophy, as well as the presence of rod-shaped particles, presumed to be baculovirus, in the nuclei (Figs 2,3 ). Such pathological changes were common and always observed in the diseased larvae from the culture farms at both Ube and Aio.

Abundant clusters of these particles were found in the lumen of the affected organ. No such changes or rod-shaped virus particles were seen in the control preparations of normal larvae.

As shown in Figure 2, the virions contained rod-shaped nucleocapsids (arrow) and were enveloped twice with outer and inner membrane as an essential structural component (insert). Sometimes the envelopes were convex or concave. The average length and diameter of virions were $310 \mathrm{~nm}$ and $72 \mathrm{~nm}$, respectively. The average length of the nucleocapsids was $250 \mathrm{~nm}$ in longitudinal section and the average diameter of the nucleocapsids was $36 \mathrm{~nm}$. The average diameter of convex envelopes or protruded envelopes was $130 \mathrm{~nm}$. Incomplete viral particles representing virogenic stages were found in the affected nuclei. A fibrillar structure which might represent virogenic materials was also observed (Fig. 2). The latter structure was similar to stromal fibrils revealed in the nuclei of fat-body cells in "Malaya disease" of an insect Oryctes rhinoceros reported by Huger (1966). In general, the virion consisted of one rod-shaped nucleocapsid within a single envelope. However, we rarely encountered virions with two nucleocapsids enclosed within a single envelope (Fig. 4). The spirally arranged capsomeres were at a 37 to $38^{\circ}$ angle to a horizontal line meeting at right angles with the long axis of the virion (Fig. 5).

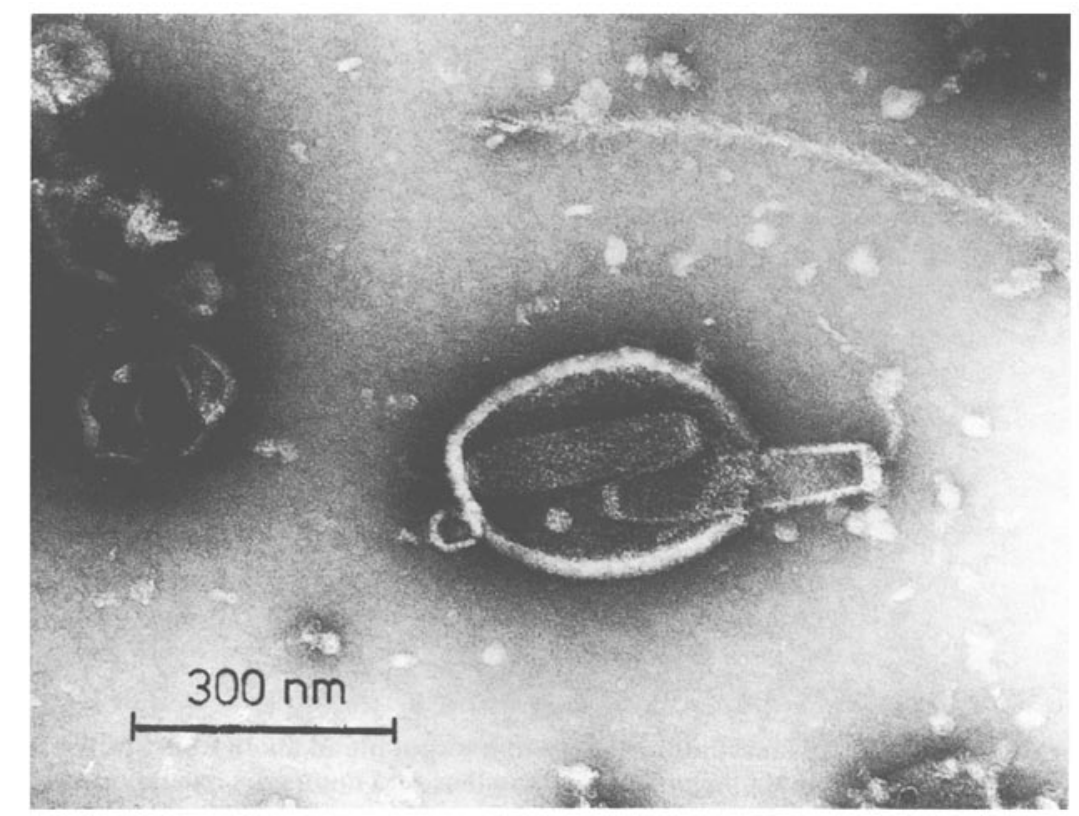

Fig. 4. Electron micrograph of negatively stained virion. Two nucleocapsids are enclosed within a single envelope which is partly broken. One nucleocapsid is protruding 


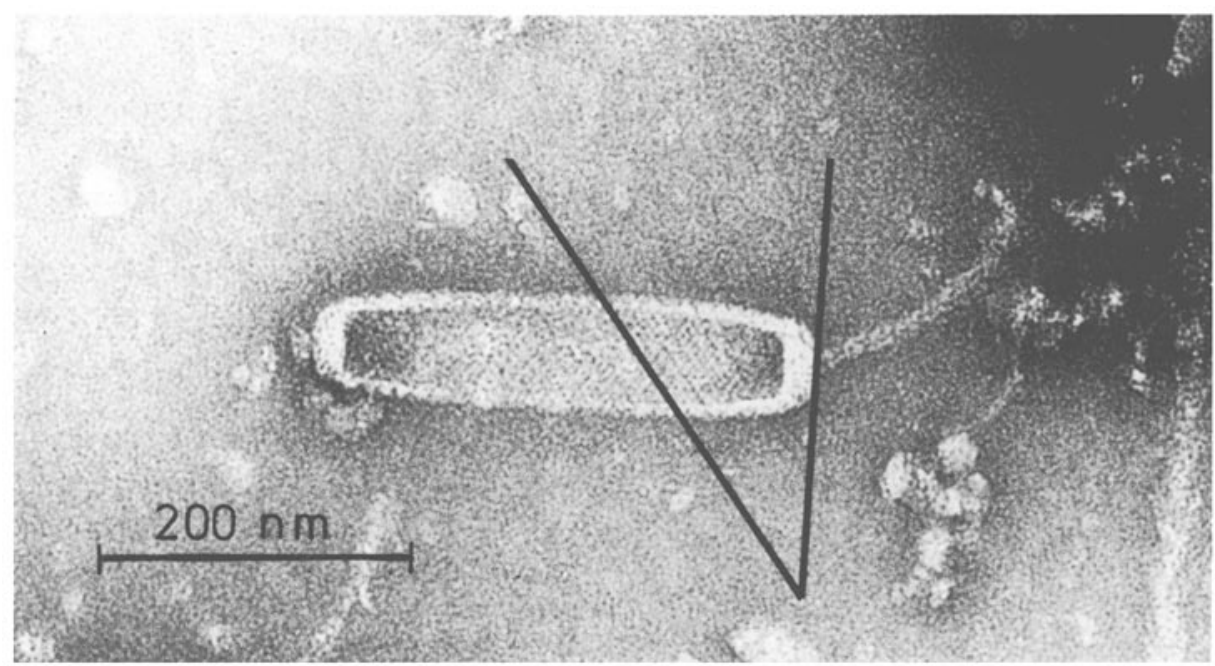

Fig. 5. Electron micrograph of negatively stained nucleocapsid. The spirally arranged capsomeres are at an angle of 37 to $38^{\circ}$ to a horizontal line meeting at right angles with the long axis of the nucleocapsid

The results obtained from oral inoculation of the 2nd post-larval stage are presented in Table 1. While the controls exhibited no mortality, the inoculation group showed that cumulative mortality was $83 \%$ up to 4 days after inoculation and revealed abnormal nuclei in the mid-gut gland $(81 \%)$ and in the intestine $(43.7 \%)$, whereas no cellular changes were observed in the control group. The results obtained from waterborne inoculation of the mysis are shown in the same table. While cumulative mortality in the experiment up to 4 days post-inoculation, was $51.1 \%$, mortality in the control was $9.1 \%$ over the same period. Additionally, there were differences between average body length of shrimps from the experimental group and the control group. The results obtained from waterborne inoculation of juveniles at the 9th post-larval stage showed acute infection, but no mortality occurred during this experimental period.

Table 1. Results obtained from infectivity experiments. Control in brackets

\begin{tabular}{|c|c|c|c|c|}
\hline \multirow[b]{2}{*}{$\begin{array}{l}\text { Day after } \\
\text { inoculation }\end{array}$} & \multicolumn{4}{|c|}{ Cumulative mortality $(\%)$} \\
\hline & \multicolumn{2}{|c|}{$\begin{array}{l}\text { Waterborne inoculation } \\
\text { of mysis II or III }\end{array}$} & \multicolumn{2}{|c|}{$\begin{array}{l}\text { Oral inoculation of juveniles } \\
\text { at } 2 \text { nd post-larval stage }\end{array}$} \\
\hline 2 & \multicolumn{2}{|l|}{$6(6)$} & \multicolumn{2}{|c|}{$30(0)$} \\
\hline 3 & \multicolumn{2}{|l|}{$39.1(8.5)$} & \multicolumn{2}{|c|}{$60(0)$} \\
\hline 4 & \multicolumn{2}{|l|}{$51.1(9.1)$} & \multicolumn{2}{|c|}{$83(0)$} \\
\hline & \multicolumn{2}{|c|}{$\begin{array}{l}\text { Difference in average body } \\
\text { length }(\mathrm{mm}) \text { of survivors: }\end{array}$} & \multicolumn{2}{|c|}{$\begin{array}{l}\text { Incidence of abnormal } \\
\text { nuclei }(\%) \text { in survivors }\end{array}$} \\
\hline & Experimental group & 4,96 & Mid-gut & 81 \\
\hline & Control group & 6.05 & Intestine & 43.7 \\
\hline
\end{tabular}




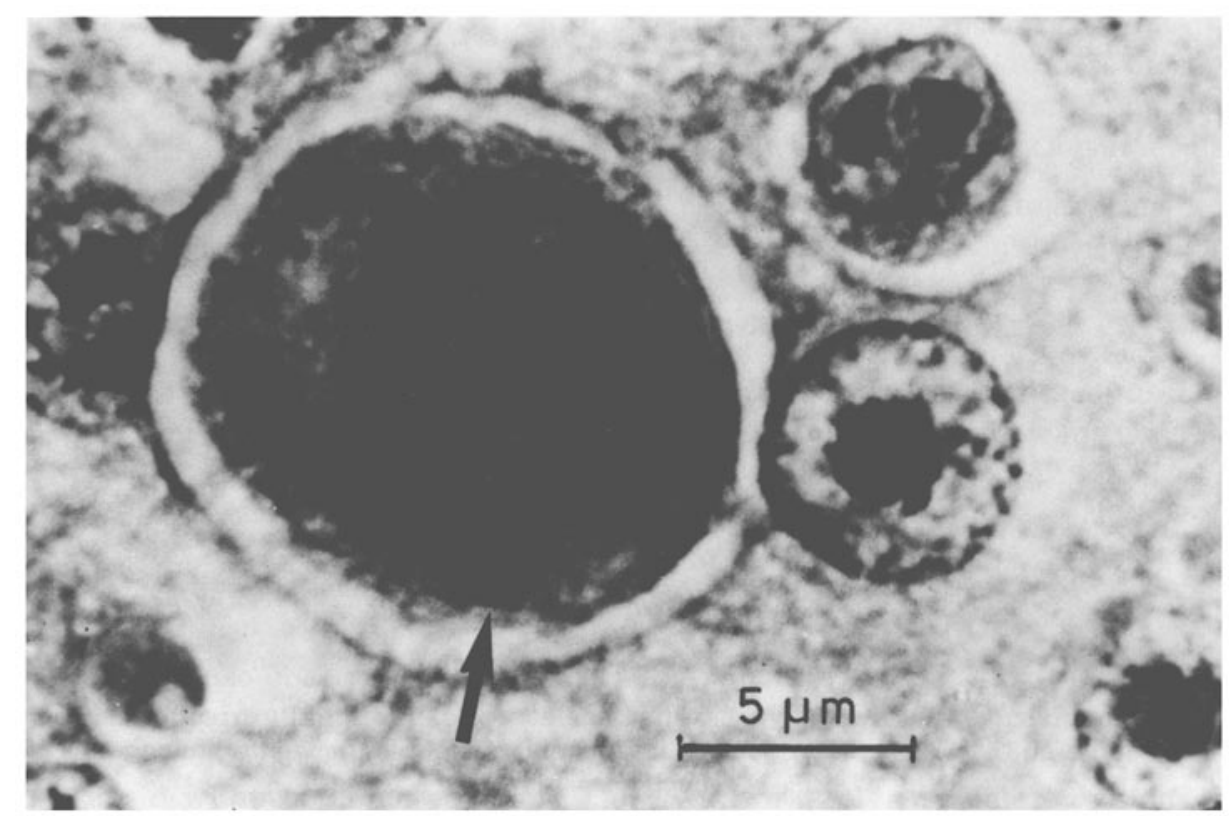

Fig. 6. Squash and stained preparation of spontaneously affected mid-gut gland (hematoxylin and eosin). Note the hypertrophied nuclei (arrow)

Ultrathin sections of the gland infected, following waterborne inoculation of the 2nd post-larval stage, revealed nuclear hypertrophy and severe infection accompanied by numerous baculoviruses (Fig. 3). These results were not different from those obtained from the spontaneously affected shrimp larvae concerning histological alterations.

Squashed and stained preparations of the affected mid-gut gland indicated hypertrophied nuclei (Fig. 6). In addition, it was demonstrated that indirect immunofluorescence technique can be used to detect infected stages $24 \mathrm{~h}$ after inoculation.

\section{DISCUSSION}

Baculoviruses comprise the following subgroups (Matthews, 1982): Baculoviruses of subgroup $A$ and $C$ replicate exclusively in the nucleus; those of subgroups $B$ replicate largely in the nucleus, but replication can occur in the cytoplasm. Virions may be occluded or non-occluded, depending upon the time or sequence in the infection cycle or the virus species. Virions of subgroups A and B can be occluded in the crystalline protein occlusion body, which may be polyhedral in shape and contain many virus particles (subgroup A), or be ovicylindrical and contain only one or rarely two particles (subgroup B). Subgroup $C$ viruses have no occlusion bodies. Consequently, the baculovirus we found in kuruma shrimp could be a member of subgroup $C$ (non-occluded rod-shaped nuclear viruses).

Three members of Baculoviridae have been identified in Penaeus thus far (see Table 2). The agent described in this paper is different from Baculovirus penaei which was identified and named after $P$. duorarum and $P$. aztecus (Couch, 1974, 1975, 1976; 


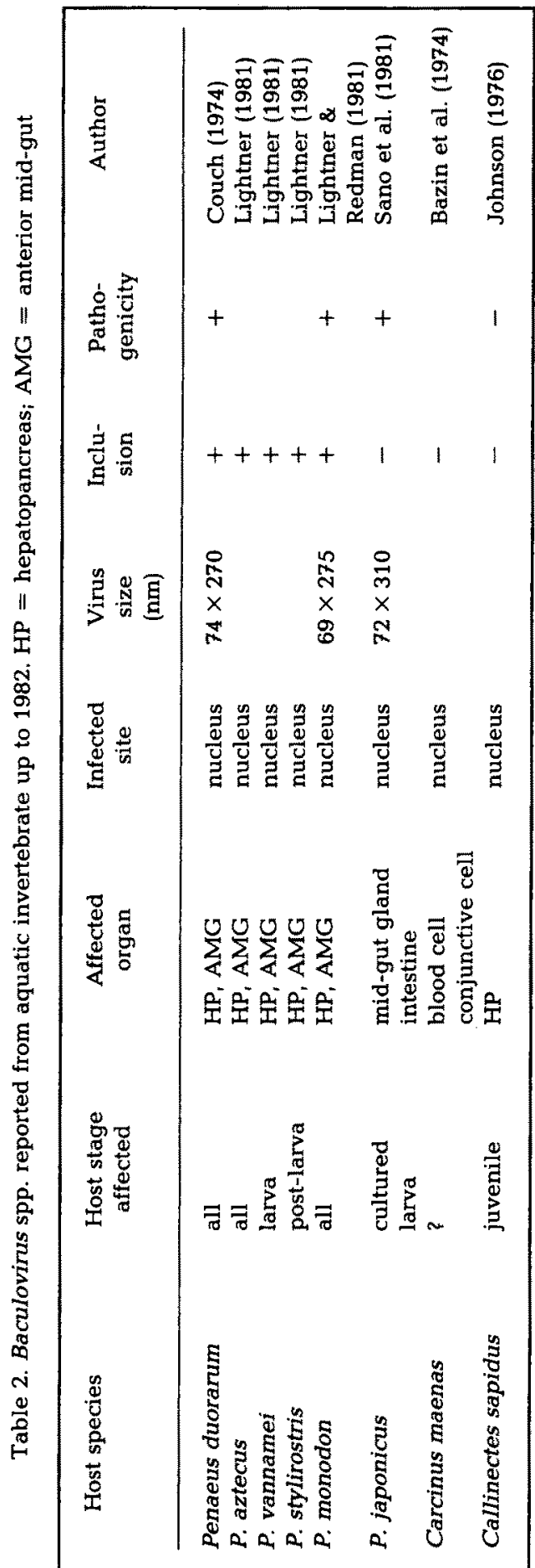


et al., 1975); it is also different from baculovirus found in P. monodon by Lightner \& Redman (1981). They produced characteristic nuclear occlusions. On the other hand, such inclusion bodies were not observed in the affected mid-gut gland cells of kuruma shrimp.

According to Bonami (pers. comm.), there are two species or types of baculovirus found in European marine invertebrates. In these two, the spirally arranged capsomeres were at $0^{\circ}$ and a 37 to $38^{\circ}$ angle, respectively, to a horizontal line meeting at right angles with the long axis of the virion. The virus considered here was at the latter angle.

We have tried to establish the shrimp cell line, but have not succeeded thus far. Although the virus has not yet been propagated in culture, three different infectivity experiments made in this study indicated that this epizootic is almost certainly a viral infection. It is suggested that viruses released with feces into the water of intensive culture systems of kuruma shrimp play an important role in the horizontal transmission. As Table 1 shows, this baculovirus, following the very acute infection and short incubation period, brings on severe mortality of kuruma shrimp in the post larval stage.

Evidence could be provided that hypertrophied nuclei observed in squashed and stained preparations of the affected mid-gut gland are a reliable presumptive diagnostic indication for BMN. Also, the indirect immunofluorescence technique can be applied to detect the infection at an early stage; it can be used as a confirmative diagnostic technique.

\section{LITERATURE CITED}

Bazin, F., Monsarrat, P., Bonami, J. R., Croizier, G., Mernadier, G., Quiot, J. M. \& Vago, C., 1974. Particules virales de type Baculovirus observées chez le crabe Carcinus maenas, - Revue Trav. Inst. Pêch. marit. 38, 205-208.

Couch, J. A., 1974. Free and occluded virus similar to Baculovirus in hepatopancreas of pink shrimp. - Nature, Lond. 247, 229-231.

Couch, J. A., 1975. An enzootic nuclear polyhedrosis virus of pink shrimp: ultrastrucuture, preva* lence and enhancement. - J. Invertebr. Pathol. 24, 311-331.

Couch, J. A., 1976. Attempts to increase baculovirus prevalence in shrimp by chemical exposure.-Prog. Exp. Tumor Res, 20, 304-314.

Couch, J. A., Summer, M. D. \& Cowtney, L., 1975. Environmental significance of baculovirus infections in estuarine and marine shrimp. - Ann. N. Y. Acad. Sci. 266, 528-536.

Huger, A. M., 1966. A virus disease of the Indian rhinoceros beetle, Oryctes rhinoceros (Linnaeus), caused by a new type of insect virus, Rhabdinovirus oryctes gen. n., sp. n. - J. Invertebr. Pathol. $8,38-51$.

Johnson, P. T., 1976. A baculovirus from the blue crab, Callinectes sapidus. - Proc. int. Colloq, Invertebr. Pathol, 1, 24.

Lightner, D. V. \& Redman, R. M., 1981. A Baculovirus-caused disease of the penaeid shrimp, Penaeus monodon. - J. Invertebr. Pathol. 38, 299-302.

Lightner, D. V., 1981. Diseases of cultured penaeid shrimp: Pre-print of a review paper. In: CRC handbook of aquaculture. Ed. by J. McVey. The Univ. Arizona, Tucson, Arizona, 1-67.

Matthews, R. E. F., 1982. Classification and nomenclature of viruses. - Intervirol. 17, 52-54.

Sano, T., Nishimura, T., Ogura, K., Momoyama, K. \& Takeno, N., 1981. Baculovirus infection of kuruma shrimp, Penaeus japonicus in Japan. - Fish. Pathol. 15, 185-191.

Vago, C., Amargier, A., 1963. Coloration histologique pour la différenciation des corps d'inclusion polyèdriques de virus d'insectes. - Annls. Epiphyt. 14, 269-274. 